Please cite this article as:

Heyvaert, M., \& Onghena, P. (2014). Analysis of single-case data: Randomisation tests for measures of effect size. Neuropsychological Rehabilitation, 24, 507-527.

doi:10.1080/09602011.2013.818564 


\title{
Analysis of single-case data: Randomisation tests for measures of effect size
}

\author{
Mieke Heyvaert \& Patrick Onghena \\ Faculty of Psychology and Educational Sciences \\ KU Leuven, Belgium
}

Author Note:

Mieke Heyvaert is a Postdoctoral Fellow of the Research Foundation - Flanders (Belgium).

Correspondence concerning this article can be addressed to Dr. Mieke Heyvaert,

Methodology of Educational Sciences Research Group, Andreas Vesaliusstraat 2 - Box 3762,

B-3000 Leuven, Belgium. Phone +32 16 326265. Fax +32 16326200.

E-mail Mieke.Heyvaert@ppw.kuleuven.be 
Abstract: Single-case experiments can be used to evaluate the effect of an intervention or treatment for a single entity. The internal validity and statistical conclusion validity of singlecase experiments can be improved by incorporating randomization in their design. In this article, we explain how to design randomized single-case phase and alternation designs as well as randomized simultaneous and sequential replication designs, and how to conduct randomization tests for those designs. Advantages and limitations of randomization tests are discussed. In order to not only determine the (non)randomness of an intervention effect, but also the magnitude of this effect, we propose to use an effect size index as a test statistic for the randomization test. We illustrate this combination for the design and analysis of an $A B A B$ phase design, using a free software package.

Keywords: single-subject experimental design (SSED); single-case experimental design (SCED); randomization test; nonparametric statistical significance test; statistical software 


\section{RANDOMISATION TESTS FOR SINGLE-CASE EXPERIMENTAL DESIGNS}

Single-case experiments (SCEs) can be used to evaluate the effect of an intervention or treatment for a single entity: the intervention or treatment can be considered as one of the levels of the independent variable, which is manipulated by the experimenter, and the effect can be evaluated by a dependent variable, which is measured repeatedly for this entity over time. In research on rehabilitation, recovery, and brain plasticity, SCEs are often used to examine the effectiveness of an intervention for a single person or a limited number of persons (e.g., McKerracher, Powell, \& Oyebode, 2005; Rasquin, van de Sande, Praamstra, \& Van Heugten, 2009; Samuel et al., 2000).

The internal validity and statistical conclusion validity of SCEs can be improved by incorporating randomization in their design (Edgington \& Onghena, 2007; Kratochwill \& Levin, 2010; Onghena \& Edgington, 2005). The randomization procedure involves the random assignment of the measurement times of the SCE to baseline and treatment, before the start of the SCE. Randomization can strengthen the internal validity of SCEs because it yields statistical control over known and unknown confounding variables (Levin \& Wampold, 1999; Onghena, 1992). As such, it enhances the scientific credibility of SCEs. Additionally, randomization can enhance the statistical conclusion validity of SCEs because it leads to a statistical test based on the randomization as it was realized in the SCE design: the randomization test (RT) (Kratochwill \& Levin, 2010; Onghena, 2005).

RTs can be used to rule out the null hypothesis that there is no differential effect of the levels of the independent variable on the dependent variable (Onghena \& Edgington, 2005). In order to use RTs, a number of steps have to be conducted. We have to formulate the null hypothesis and alternative hypothesis, specify the level of significance $\alpha$, and designate the number of measurement times. Additionally, an appropriate test statistic should be selected: the test statistic has to be chosen in accordance to the kind of effects we expect or predict. 
Using an RT requires that we design the SCE so that it involves random assignment of the observation occasions to the levels of the independent variable. All assignments that are possible based on this randomization schedule should be recorded (cf. 'randomization referral approach' discussed in Edgington \& Onghena, 2007). Then one of these possible assignments is randomly chosen: our SCE will follow this assignment. Afterwards, we can run our SCE, collect the data, and calculate the test statistic for the obtained data. Subsequently, we can construct the randomization distribution: we look at all possible assignments (or a random subset of all possible assignments for the application of a Monte Carlo RT, see Onghena \& Edgington, 2005) and calculate the chosen test statistic for each of these assignments. The randomization distribution has the same function as the sampling distribution in classical tests. However, in order to construct the randomization distribution only the random assignment as it was actually implemented while carrying out the study is taken into account, because this assignment procedure is the stochastic foundation for the reference distribution of an RT. In all possible assignments for which the test statistic is computed, the exact sequence of the measurements actually obtained remains intact; only the levels of the independent variable (the 'treatment labels') are redistributed or reshuffled to generate the virtual replications. Finally, we can determine the statistical significance of our observed test statistic by examining its position within the distribution of all possible test statistic values. The $p$ value of the RT is calculated as the proportion of possible test statistic values that is as extreme, or even more extreme, than the observed test statistic.

There are two main types of designs for SCEs: phase designs and alternation designs. The randomization procedure for single-case phase designs involves the random determination of the moments of phase change. For single-case alternation designs, the treatment order is randomly determined. 


\section{Randomization tests for single-case phase designs}

In single-case phase designs all measurement times are divided into phases and several consecutive measurements are taken in each phase (Edgington, 1975, 1980; Onghena, 1992). Examples of single-case phase designs are A-B designs, A-B-A designs (i.e., withdrawal or reversal designs), A-B-A-B designs, A-B-A-C-A designs (to compare separate treatments or components), A-B-A-B-BC-B-BC designs (to assess the interactive effects of the treatments or components), A-B-A-B-B1-B2-...BN designs (to examine specific variations of the treatment procedure), and changing criterion designs (cf. Barlow, Nock, \& Hersen, 2009).

How can we introduce randomization in single-case phase designs in order to enhance their internal validity? For single-case phase designs the order of the phases is fixed, so the only random element that can be introduced, refers to the moments of phase change. For instance, in an $\mathrm{ABAB}$ design the moment when the first intervention phase, the second baseline phase, and the second intervention phase start, can be randomly determined. When designing a randomized single-case phase design, the total number of available measurement times and the number of phases should be decided a priori. Additionally, the possible moments of phase change should be decided a priori. An efficient way to determine and list all possible patterns of phase changes is to fix the minimum lengths of the phases (i.e., the minimum number of measurement times for each phase). For instance, following the What Works Clearinghouse Standards (Kratochwill et al., 2010) a researcher who wants to conduct a randomized single-case $\mathrm{ABAB}$ phase design (i.e., 4 phases) with 22 measurement times, can decide that there should be minimally 5 measurement times for each phase. These are the ten possible assignments for this design:

AAAAABBBBBAAAAABBBBBBB

AAAAABBBBBAAAAAABBBBBB

AAAAABBBBBAAAAAAABBBBB 
AAAAABBBBBBAAAAABBBBBB

AAAAABBBBBBAAAAAABBBBB

AAАAАВBВBВBВАААAАВВBВB

AAAAAABBBBBAAAAABBBBBB

AAAAAABBBBBAAAAAABBBBB

AAAAAABBBBBBAAAAABBBBB

AAAAAAABBBBBAAAAABBBBB.

\section{Randomization tests for single-case alternation designs}

If any level of the independent variable could be present at each measurement time (i.e., quick and repeated alternation of treatments is possible) within a single subject, one can use a single-case alternation design (Edgington, 1967; Onghena \& Edgington, 1994). We refer to the eighth chapter of Barlow et al. (2009) for an excellent discussion of terminology, procedural considerations, advantages, and examples of single-case alternation designs.

We can introduce randomization in single-case alternation designs by randomly ordering the levels of the independent variable. In completely randomized alternation designs, the number of possible assignments is determined by taking into account the number of levels of the independent variable and the number of measurement times for each level. For instance, there are 20 possible assignments for a single-case alternation design with 2 levels (A and B) and 3 measurement times for each level: AAABBB, AABABB, AABBAB, AABBBA, ABAABB, ABABAB, ABABBA, ABBAAB, ABBABA, ABBBAA, BAAABB, BAАВАВ, ВААВВА, ВАВААВ, ВАВАВА, ВАВВАА, ВВАААВ, ВВААВА, ВВАВАА, and BBBAAA. In a completely randomized alternation design 1 of these 20 possible assignments is randomly selected: the SCE will follow this assignment. 
When certain assignments of a completely randomized alternation design are undesirable (e.g., assignments with three successive identical treatments), other alternation designs can be applied. The first alternative is the randomized block alternation design: we pair each $\mathrm{AB}$ sequence and randomly determine the order of the two members for each pair. For the previous example, this results in eight possible assignments: $\mathrm{ABABAB}, \mathrm{ABABBA}$, ABBAAB, ABBABA, BABABA, BABAAB, BAABBA, and BAABAB. However, not only the six assignments with three successive identical treatments (AAABBB, AABBBA, ABBBAA, BAAABB, BBAAAB, BBBAAA) are no longer generated as possible assignments by the randomized block alternation design. Six other assignments as well are no longer generated: AABABB, AABBAB, ABAABB, BABBAA, BBAABA, and BBABAA. Because, based on the number of possible assignments, the randomized block alternation design can be considered as being too restrictive, a second alternative to the completely randomized alternation design was developed: a randomized version of the alternating treatments design along with an algorithm to enumerate and randomly sample the set of all acceptable assignments (Onghena \& Edgington, 1994). This second alternative only excludes the 6 assignments with 3 successive identical treatments and gives us all 14 acceptable assignments.

\section{Replication designs}

As discussed above, the internal validity and statistical conclusion validity of SCEs can be improved by incorporating randomization in their design. The external validity or generalizability of SCEs can be enhanced by using systematic within- or between-subjects replication. The extent to which an intervention's effect is similar across replications helps researchers to establish the external validity of the intervention (Kratochwill \& Levin, 2010). 
We differentiate between simultaneous and sequential replication designs (cf. Onghena \& Edgington, 2005).

\section{Simultaneous replication designs}

In simultaneous replication designs, the replications are carried out concurrently. The multiple baseline design (MBD) is an often applied simultaneous replication design (cf. Barlow et al., 2009; Kazdin, 2011). In a multiple baseline across participants design, several persons are included. Often, an $\mathrm{AB}$ phase design is carried out for each individual, with different starting points for the intervention phase. Because the data are collected concurrently for all persons, historical confounding variables can be ruled out when there are no changes that correspond across the data series of the included persons (Christ, 2007). If a phase change is implemented for one person and produces a change in the level of the dependent variable for that person, while little or no change is observed for the other persons, then it is less likely that other external events are responsible for the change in the dependent variable, and more likely that the independent variable is responsible for the change.

Randomization can be introduced in simultaneous replication designs by separately implementing randomization schedules in the designs for the included participants (Onghena, 1992). A potential limitation of the procedure is that exactly the same randomization schedule could be selected for each participant and thus the design would not have the temporal staggering that is desired by researchers using MBDs. As a solution, Koehler and Levin (1998) proposed to impose a restriction that ensures temporal staggering: 'regulated randomization'. Solutions are also included in free software packages for designing and analyzing SCEs. For instance, in the Single-Case Data-Analysis (SCDA) package from Bulté and Onghena (2009), different possible starting points for the intervention phase can be inserted for each participant when designing randomized MBDs (i.e., no overlap between the 
possible starting points of the different participants). The RT for simultaneous replication designs is based on the randomization in each of the separate designs and uses a multivariate statistic to test the null hypothesis that there is no effect for any of the participants (Onghena \& Edgington, 2005).

\section{Sequential replication designs}

In sequential replication designs, the replications are implemented consecutively. Because the replicated SCEs can be considered as separate studies, they can be analyzed by meta-analytic procedures (Onghena, 2005). A first group of procedures focuses on combining the $p$ values of the separate SCEs. Examples are the Stouffer procedure (cf. Hedges, Cooper, \& Bushman, 1992; Rosenthal, 1978), the additive procedure (Edgington, 1972; Onghena \& Edgington, 2005), the multiplicative procedure (Edgington, 1972; Pesarin, 2001; Rosenthal, 1978), and the iterative procedure (Pesarin, 2001; Pesarin \& Salmaso, 2010).

A second group of procedures focuses on combining effect size indices calculated for the separate SCEs. Several authors provided overviews and comparisons of effect size indices for SCEs (e.g., Brossart, Parker, Olson, \& Mahadevan, 2006; Campbell, 2004; Maggin et al., 2011; Manolov, Solanas, \& Leiva, 2010; Manolov, Solanas, Sierra, \& Evans, 2011; Parker \& Brossart, 2003; Parker \& Hagan-Burke, 2007; Parker, Vannest, \& Davis, 2011; Wolery, Busick, Reichow, \& Barton, 2010). A 'basic' procedure for combining effect sizes of SCEs is taking the (weighted) average. For example, in his meta-analysis of SCEs on the efficacy of behavioral interventions for reducing problem behavior in persons with autism, Campbell (2003) calculated three effect sizes (i.e., percentage of nonoverlapping data, percentage of zero data, mean baseline reduction) for each participant and article. Within each article, effect sizes were weighted according to the number of data points per participants, and then averaged for all participants to yield three effect sizes per article. In order to combine the 
effect sizes per study over all the included studies, one can simply average the calculated effect sizes. However, it is also possible to calculate an overall weighted average, with the weight depending on the number of participants included in each study. More advanced procedures are for instance the three procedures described by Busk and Serlin (1992), hierarchical linear modeling (HLM; Van den Noortgate \& Onghena, 2003a, 2003b, 2008), and a recently developed standardized mean difference effect size for SCEs that is directly comparable with Cohen's $d$ (Hedges, Pustejovsky, \& Shadish, 2012). We refer to the article of Shadish, Rindskopf, and Hedges (2008) for a discussion of the more advanced procedures for parametric and nonparametric effect size estimation and regression approaches for the meta-analysis of SCEs.

\section{ANALYSIS OF SINGLE-CASE DATA}

\section{Visual analysis}

We argue that the combined use of visual analysis, RTs, and effect sizes is necessary for a comprehensive analysis of randomized SCEs. Visual analysis can be used for an initial assessment of the intervention effects by examining six features of graphed SCE data: level, variability, trend, immediacy of effect, overlap, and consistency of data patterns across similar phases (Bulté \& Onghena, 2012; Kazdin, 1982; Kratochwill et al., 2010). Advantages of visual analysis are that it is quick, easy, and inexpensive to use, and that it is widely accepted and understood. Major drawbacks are the low average interrater agreement, and thus the low reliability or consistency (e.g., DeProspero \& Cohen, 1979; Harbst, Ottenbacher, \& Harris, 1991; Matyas \& Greenwood, 1990a, 1990b; Ottenbacher, 1990; Park, Marascuilo, \& GaylordRoss, 1990) and the low accuracy because of the inflated Type 1 error rates (e.g., Borckardt, Murphy, Nash, \& Shaw, 2004; Ferron \& Jones, 2006; Matyas \& Greenwood, 1990a). 
Furthermore, Kazdin $(1982,2011)$ describes several situations in which visual analysis is inadequate and additional statistical analyses are necessary (e.g., no stable baseline, increased intrasubject variability, weak or ambiguous effects, small but important and reliable changes, when an SCE is implemented in the natural environment so that it is necessary to statistically control for extraneous factors).

\section{Randomization tests}

Statistical significance tests such as RTs can be used to respond to the drawbacks of visual analysis of SCE data. For instance, statistical significance tests produce consistent results that are independent of the performer of the analysis, they can be applied with unstable baseline data and with increased intrasubject variability, and powerful statistical tests have the ability to detect small but systematic treatment effects that might be ignored by visual analysis (Kazdin, 2011; Nourbakhshs \& Ottenbacher, 1994).

In comparison with most parametric tests, an advantage of RTs is that they are valid for SCEs without making distributional assumptions (Edgington, 1967, 1980; Edgington \& Onghena, 2007). For instance, RTs can provide valid $p$ values even if there are severe outliers, if the variances are highly unbalanced, or if one of the variances is zero (Edgington \& Onghena, 2007; Onghena \& Edgington, 2005). An advantage of RTs to other nonparametric tests, such as the Wilcoxon-Mann-Whitney test and the Kruskal-Wallis test, is that the $p$ value can be derived without degrading the observed scores to ranks (Onghena \& Edgington, 2005).

Another advantage of using RTs is that it is a flexible approach that can be used for even the most complex SCE designs (Edgington, 1984; Levin \& Wampold, 1999; Onghena, 1992). Additionally, RTs allow much flexibility in defining the test statistic. SCE researchers can customize RTs so that they are sensitive to the specific effect they anticipate, such as changes in level, changes in trend, changes in both level and trend, and changes in variability 
(Ferron \& Sentovich, 2002). It is very appealing that the choice of the test statistic for the RT is not confined to statistics for which the sampling distribution has been derived or tabulated (Onghena \& Edgington, 2005). Another advantage of using RTs is that they are easy to apply: the underlying logic of RTs is straightforward and software has been developed to increase the user-friendliness of RTs for SCEs (e.g., Bulté \& Onghena, 2008, 2009; Koehler \& Levin, 2000; Todman \& Dugard, 2001). Furthermore, as discussed above, randomization not only provides the foundation for a valid statistical test, but also offers a solution to internal validity threats for SCEs (Kratochwill \& Levin, 2010).

A disadvantage of the use of RTs for SCEs might be that, in some circumstances, the statistical power of RTs is compromised, and so that small but systematic treatment effects are missed, just like with visual analysis. Another disadvantage is that RTs can only be used when observation occasions can be randomly assigned to the experimental treatments. This will be further elaborated in the Discussion section.

\section{Effect size measures}

Several leading organizations emphasize the importance of reporting effect sizes for primary outcomes (e.g., American Psychological Association, 2010; Kratochwill et al., 2010; Task Force on Evidence-Based Interventions in School Psychology, 2003). For SCEs, effect size measures are used to quantify the amount of behavior change between baseline and intervention phases. However, so far there are no agreed-upon methods or standards for effect size estimation for SCEs (Kratochwill et al., 2010). Different effect size indices are appropriate for different SCE designs and data patterns (Gage \& Lewis, 2013; Manolov et al., 2011).

Standardized mean difference (SMD) approaches have been used to describe the magnitude of an effect for SCEs (cf. Busk \& Serlin, 1992). Additionally, several parametric 
and nonparametric methods to calculate effect size indices for SCEs have been developed. Examples of parametric methods are piecewise regression (Center, Skiba \& Casey, 19851986), hierarchical linear modeling (Van den Noortgate \& Onghena, 2003a, 2003b, 2008), generalized least squares (Maggin et al., 2011), and using interrupted time series analyses to derive an estimate of the intervention effect (ITSA; e.g., Crosbie, 1993; Hartmann et al., 1980; Jones, Vaught, \& Weinrott, 1977).

Percentage of Nonoverlapping Data (PND; Scruggs, Mastropieri, \& Casto, 1987) is the most often applied nonparametric effect size index for SCE research, and so presumably the most familiar effect size index to the reader. Advantages of PND are that is correlates very well with visual analysis judgments, that it can easily be calculated directly from graphical displays, that it is easily understood and interpreted by practitioners, and that it has a high inter-rater reliability (cf. Scruggs \& Mastropieri, 2013). Limitations of this index are that PND (a) is likely to erroneously represent effects when outliers are present in the baseline phase, (b) when the treatment has a detrimental effect, (c) when trend is present in the data, and (d) that PND is not able to discriminate satisfactorily between SCE designs that produce no overlap yet show large differences in the distance between treatment and baseline data points (Allison \& Gorman, 1993; Campbell, 2013). Several alternative effect size indices have been developed to address the drawbacks of PND, such as IRD (Improvement Rate Difference), NAP (Nonoverlap of All Pairs), PAND (Percentage of All Nonoverlapping Data), PEM (Percentage of Datapoints Exceeding the Median), PNCD (Percentage of Nonoverlapping Corrected Data), PZD (Percentage of Zero Data), SLC (Slope and Level Change), and Tau- $U$ (Tau for Nonoverlap with Baseline Trend Control) (cf. Brossart et al., 2006; Campbell, 2004; Maggin et al., 2011; Manolov et al., 2010, 2011; Parker \& Brossart, 2003; Parker \& Hagan-Burke, 2007; Parker et al., 2011; Wolery et al., 2010 for discussions and overviews). 


\section{Randomization tests for measures of effect size}

In order determine the (non)randomness of an intervention effect as well as the magnitude of this effect, we recommend to complement the results of an RT with an effect size measure. One can separately conduct an RT and calculate an effect size index, and report them both when publishing an SCE. However, it is also possible to directly combine them by using an effect size index as a test statistic in the RT. As such, the effect size for the observed SCE data can be calculated and this value can be located in a distribution of values that were equally likely if the null hypothesis of no differential effect of the levels of the independent variable on the dependent variable is true (Onghena \& Edgington, 2005).

It is argued that of all the nonparametric effect size indices, only PND lacks a known sampling distribution, which prevents the calculation of $p$ values (Parker et al., 2011). However, by using PND as the test statistic for the RT, we do can determine the statistical significance of the observed PND value.

\section{Randomization tests for measures of effect size: An example}

Let us look at an example of how to design randomized SCEs and how to analyze them using RTs for a measure of effect size. Suppose that we are interested in evaluating a new treatment program for decreasing the post-traumatic stress symptoms of a participant, called Tim. We want to compare the effects of the old treatment program with the new program using a randomized single-case $\mathrm{ABAB}$ phase design (with $\mathrm{A}=$ old treatment program; $\mathrm{B}=$ new treatment program). We will compare both programs during a period of 27 weeks. Each week, we will measure the total score on the Impact of Event Scale (IES; Horowitz, Wilner, \& Alvarez, 1979). The IES is often used to assess post-traumatic stress symptoms: on the original 15 -item IES the total scores have a possible range of 0 to 75 , with higher scores indicating a greater frequency of intrusive thoughts and attempts at avoidance 
(Joseph, 2000). Our SCE consists of four phases, and we want at least five measurements per phase. The three moments of phase change are randomly determined before the start of the experiment. We will use the SCDA package from Bulté and Onghena $(2008,2009,2012)$ to design our SCE and analyze the data. RcmdrPlugin.SCDA is an R Commander plugin for designing and analyzing SCEs. This package provides a Graphical User Interface for the Single-Case Visual Analysis (SCVA), Single-Case Randomization Test (SCRT) and SingleCase Meta-Analysis (SCMA) packages.

The null hypothesis for our RT says that Tim's responses (i.e., weekly total score on IES) are independent of the treatment (i.e., old versus new treatment program) under which they are observed. The alternative hypothesis can be formulated in a directional manner: we expect a positive effect (i.e., lower total score on IES) of the new treatment program, compared to the old treatment program. We will use .05 as the level of significance $(\alpha)$ for the RT.

We choose PND as the test statistic for the RT in this example, because of several reasons. First of all, we mentioned that PND is the most often applied nonparametric effect size index for SCE research, and so presumably the most familiar effect size index to the reader. It is also a very intuitively appealing and straightforward measure of effect size that does not ask for much further clarification: the user simply has to calculate the proportion of data points in the treatment phase that exceeds the most extreme value in the baseline phase. The goal of the present paper is to present a preliminary idea of how it is possible to combine RTs and effect sizes for analyzing SCEs. For this preliminary example, we therefore select PND as a test statistic for the RT. Second, we mentioned that of the nonparametric effect size indices, only PND lacks a known sampling distribution, which prevents the calculation of $p$ values (Parker et al., 2011). However, by using PND as the test statistic for the RT, we are able to determine the statistical significance of the observed PND value. 
We emphasize that it is possible to use any effect size index as a test statistic for the RT. In the section Effect size measures, we mentioned several more recently developed overlap statistics, like NAP and PAND, which seem to overcome some of the shortcomings of PND as a descriptive measure of effect and could potentially have more power as a test statistic for an RT. However, as discussed by for instance Shadish and colleagues (2008), these recently developed overlap statistics are in need of continued improvement. Future research is needed to further develop appropriate effect size indices for SCEs, and to evaluate and compare these effect size indices. In addition, future research can focus on combining the more recently developed overlap statistics with RTs.

When describing the steps that have to be conducted in order to use RTs, we said that the test statistic has to be chosen in accordance to the kind of effects we expect or predict. Researchers can choose their test statistic in the same way as they would have chosen their descriptive measure to represent the data. Furthermore, researchers can choose their test statistic to maximize the power of the RT. An interesting avenue for future research would be to look at the power differences among alternative test statistic choices.

Let us now return to our example. First, we should record all assignments that are possible based on the randomization schedule. In SCDA, we click on 'SCRT', 'DESIGN YOUR EXPERIMENT', and 'NUMBER OF POSSIBLE ASSIGNMENTS'. We select 'ABAB PHASE DESIGN' as the design type, set 'NUMBER OF OBSERVATIONS' to 27, and set the limitation (i.e., the minimum number of observations per phase) to 5 . The result of this computation is 120: there are 120 possible assignments for an $\mathrm{ABAB}$ design with 27 measurement times and a minimum of 5 measurement times per phase.

Second, randomly 1 of these 120 assignments should be selected: our SCE will follow this assignment. We can conduct the random selection in SCDA: we click on 'SCRT', 'DESIGN YOUR EXPERIMENT', and 'CHOOSE 1 POSSIBLE ASSIGNMENT'. We select 
'ABAB PHASE DESIGN' as the design type and set 'NUMBER OF OBSERVATIONS' to 27 and the limitation to 5. The following assignment was selected: AAAAAABBBBBAAAAAAAAABBBBBBB. We can collect the data for our SCE following this assignment. A hypothetical data set is presented in Table $\mathbf{1}$.

\section{Insert Table 1 about here}

We have to create a text (.txt) file containing the data in order to use the R functions described further for this example. The text file should contain two columns, separated by a tab. The first column should contain the treatment labels: A1 A1 A1 A1 A1 A1 B1 B1 B1 B1 B1 A2 A2 A2 A2 A2 A2 A2 A2 A2 B2 B2 B2 B2 B2 B2 B2. The second column should contain the total IES scores for each measurement time: 494145394842363541363848 4543514347424345362933343830 32. We do not label the rows and columns. We save our text file as 'Tim.txt'.

We can use this text file to generate a graphical presentation of our data set. In the menu SCDA, we click on 'SCVA' and 'GRAPHICAL DISPLAY'. We select 'USE DATA FROM TEXT FILE' and select our text file 'Tim.txt'. We select 'ABAB PHASE DESIGN' as the design type. This results in Figure 1.

\section{Insert Figure 1 about here}

Third, we have to calculate the test statistic (PND) for our data set. We can do this in SCDA by clicking on 'SCMA' and 'CALCULATE EFFECT SIZE'. We select 'USE DATA FROM TEXT FILE' and select our text file 'Tim.txt'. We select 'ABAB PHASE DESIGN' as the design type and the effect size index 'PND (EXPECTED DECREASE)', because we 
expected lower total scores on the IES during the new treatment program, compared to the old treatment program. Our observed test statistic (PND) is $90 \%$. Following the guidelines of Scruggs, Mastropieri, Cook, and Escobar (1986) this means that the new treatment program is highly effective.

Fourth, we can construct the randomization distribution: we look at the 120 possible assignments that were recorded at the beginning of our study and calculate the chosen test statistic for each of these assignments. In order to do that, all 27 scores are kept fixed, but the three phase transitions (i.e., from $\mathrm{A} 1$ to $\mathrm{B} 1$, from $\mathrm{B} 1$ to $\mathrm{A} 2$, and from $\mathrm{A} 2$ to $\mathrm{B} 2$ ) are randomly shifted taking into account the minimum of 5 measurement times for each phase. The 120 PND values can be calculated by using SCMA. The 120 test statistics, sorted in ascending order, represent the reference distribution for the statistical test.

Finally, we determine the statistical significance of our observed test statistic by examining its position within this reference distribution. When we sort the 120 test statistics in ascending order, we see that there is no other test statistic larger than or as large as the observed test statistic (90\%). Accordingly, the $p$ value of the RT is $1 / 120=.0083$. This $p$ value is smaller than .05 , so we reject the null hypothesis that Tim's weekly total scores on the IES are independent of the treatment under which they are observed. Tim's post-traumatic stress symptoms, as measured with the IES, are significantly less frequent under the new treatment program.

We remark that the SCDA package offers the possibility to calculate the effect sizes SMD, PND, and PEM for SCE data sets (i.e., third step in the example; cf. Bulté, Van Den Noortgate, \& Onghena, 2010). However, in order to construct the randomization distribution (i.e., fourth step in the example) and determine the statistical significance of the observed test statistic (i.e., final step in the example), the SCDA package for now only includes built-in options with differences between means as test statistics (Bulté \& Onghena, 2008). As such, 
PND is not yet a built-in standard option for constructing the randomization distribution and calculating the RT's $p$ value. However, by making small adjustments to the code, other test statistics - such as PND - could easily be adopted (cf. Bulté \& Onghena, 2008).

\section{DISCUSSION}

In this article, we discussed several advantages of using RTs for the analysis of SCEs. However, there are some important limitations as well. First, the validity of RTs is only guaranteed by design when observation occasions are randomly assigned to the experimental treatments before the start of the experiment (cf. supra). Although using RTs for SCEs does not imply checking assumptions concerning the distribution of the SCE data, the assumption of random assignment should be checked. If there is no random assignment, then assumptions regarding exchangeability or random sampling should be invoked just as with any other statistical test, and the $p$ value should be interpreted very cautiously in a noncausal descriptive manner (Edgington \& Onghena, 2007). Because the randomization of some aspect of the design has to be done before the data are collected, RTs seem incompatible with the responseguided experimentation that is common in single-case research (Onghena, 1992). Accordingly, the need to incorporate randomization in the SCE design can in some cases be seen as a limitation, given that it may not be well-aligned with clinical aims. However, it is possible to combine randomization and response-guided experimentation procedures in SCEs. A researcher can decide to make the manipulation of the conditions only partially dependent on the data: by using such a 'restricted random assignment' valid significance determination by the RT procedure is still possible (Edgington, 1980). For example, a researcher conducting an $\mathrm{AB}$ phase design can a priori decide not to introduce the experimental treatment until after the baseline data show stability (cf. response-guided experimentation), but he can supplement this procedure with the random selection of the moment when the intervention phase starts, 
after baseline stability has been attained, thereby allowing for the valid use of an RT (Edgington, 1975). Similarly, Ferron and Ware (1994) discussed a method for combining randomization and response-guided experimentation procedures in $\mathrm{AB} A \mathrm{~B}$ phase designs, that allows partial control to the researcher: the researcher has to wait until the within-phase data are stabilized (cf. response-guided experimentation), and then randomly assigns the start of the intervention within a specified region.

A second possible limitation concerns statistical power. Statistical power varies depending on the design, on the test statistic, on the level of autocorrelation, on the magnitude of the effect size, on the number of observations, and on the number of possible assignments or permutations (cf. Dugard, File, \& Todman, 2012; Ferron \& Onghena, 1996; Ferron \& Sentovich, 2002; Ferron \& Ware, 1995; Levin, Ferron, \& Kratochwill, 2012; Levin, Lall, \& Kratochwill, 2011; Manolov, Solanas, Bulté, \& Onghena, 2010). For instance, if the set of potential assignments is too small, it is possible that the SCE design has too little statistical power. For example, it is easy to see that a .05 level RT has zero power if less than 20 assignments are possible (because the smallest possible $p$ value in that case would be always larger than 1/20).

Although the power of individual $\mathrm{AB}$ designs usually is very low, the combination of three or more $\mathrm{AB}$ designs in an $\mathrm{MBD}$ raises the power considerably (Onghena \& Edgington, 2005). The standards developed by the What Works Clearinghouse (Kratochwill et al., 2010) tell that an MBD should have minimally three baseline conditions (e.g., three participants, in order to have at least three attempts to demonstrate an intervention effect), six phases (e.g., an $\mathrm{AB}$ design for each of the three participants), and five data points per phase. The simulation study of Ferron and Sentovich (2002) showed that under most conditions the statistical power of RTs to detect treatment effects that are common in SCE research $(d \geq 1.5)$ is already adequate $(>0.80)$ for an MBD with 4 participants and a total of 20 measurement occasions. 
Third, a limitation of our example concerns the fact that the effect size that is computed from the realized data set is just one number, without taking uncertainty into account. We propose that future research focusses on the issue of constructing confidence intervals for effect sizes based on the RT rationale. Confidence intervals for groupcomparison studies are usually constructed by invoking distributional assumptions (Ernst, 2004). However, these assumptions are often not met for SCE data. Constructing confidence intervals for effect sizes that correspond to RTs can be realized by inverting the RT: the 95\% confidence interval contains all effect sizes for which the null hypothesis of no treatment effect cannot be rejected at the 5\% significance level. Because constructing randomization confidence intervals for effect sizes holds great promise for the applied SCE researcher, a methodological and statistical evaluation of this method can be conducted in future research.

In the past, the computational burden was a fourth possible limitation of RTs. However, free software has been developed to address this limitation, such as RegRand for calculating RTs in MBDs (Koehler \& Levin, 2000), Excel routines (Todman \& Dugard, 2001), and SCRT-R (Bulté \& Onghena, 2008, 2009). For example, SCRT-R can be used to determine the number of possible assignments for a specific design, generate all the possible assignments for a specific design, randomly select one assignment among all theoretically possible assignments, calculate the observed value of the test statistic, construct the randomization distribution, and calculate the RT's $p$ value, for single-case phase and alternation designs (Bulté \& Onghena, 2008) as well as for simultaneous (Bulté \& Onghena, 2009) and sequential (Bulté et al., 2010) replication designs. R can be downloaded freely from the CRAN website (http://cran.r-project.org/).

In addition to these limitations to the use of RTs for the analysis of SCEs, we want to emphasize the difference between statistical significance and clinical significance. RTs are statistical significance tests. As such, they can only be used as indicators of statistical 
significance, and not of clinical significance. The construct of clinical significance is also broader than visual analysis results and effect size measures: it concerns whether the intervention makes a 'real difference' to the person and/or to others with whom the person interacts in everyday life (Allison \& Gorman, 1993; Kazdin, 1999; Perdices \& Tate, 2009). Examples of measures that can be used to consider the clinical importance of the change and the impact of the intervention on the person's everyday functioning are: no longer meeting diagnostic criteria, departure from dysfunctional behavior, falling within a normative range, risk-benefit contours, and quality of life (cf. Kazdin, 1999, 2011; Schulz et al., 2002; Shakespeare, Gebski, Veness, \& Simes, 2001).

For the example of Tim, the difference between statistical and clinical significance can be clearly demonstrated. As determined by the RT, the positive intervention effect of the new treatment program on the dependent variable is not random: we concluded that Tim's posttraumatic stress symptoms are significantly less frequent under the new treatment program, in comparison to the old program. Additionally, following the determination of Scruggs et al. (1986), the new treatment program is highly effective (i.e., PND $=90 \%$; note though that the uncertainty is not considered, cf. supra). However, when we look at the data (cf. Table 1) we see that the IES scores under the old treatment program range between 39 and 51, and between 29 and 41 under the new program. Using the IES total score, Horowitz (1982) identified thresholds for low $(<8.5)$, medium (8.6-19), and high $(>19)$ symptom levels. These levels correspond to levels of clinical concern. This means that even under the new treatment program, Tim still shows post-traumatic stress symptoms that pose clinical concerns.

\section{CONCLUSION}

In this article, we explained how to design randomized single-case phase and alternation designs as well as randomized simultaneous and sequential replication designs, 
and how to conduct RTs for those designs. Advantages of RTs are that they (a) are easy to apply, (b) respond to some important drawbacks of visual analysis of SCE data, (c) are distribution-free tests, (d) can be used without degrading the observed scores to ranks, (e) can be applied in a vast array of different SCE designs, (f) allow flexibility in defining the test statistic, (g) offer a solution to internal validity threats and enhance the statistical conclusion validity of SCEs, and that (h) recently free software has been developed to design randomized SCEs and analyze the SCE data using RTs (cf. supra). Possible limitations of RTs concern (a) statistical power, and (b) that RTs can only be used when observation occasions can be randomly (or: partially random for restricted random assignment procedures) assigned to the experimental treatments.

In order to not only determine the (non)randomness of an intervention effect, but also the magnitude of this effect, we proposed to use an effect size index as a test statistic for the RT. We illustrated this combination for the design and analysis of an SCE, using a free software package. For this example, we used PND as the test statistic for the RT. However, it is possible to use any effect size index as a test statistic for the RT. Interesting avenues for future research concern (a) the further development of appropriate effect size indices for SCEs, and their evaluation and comparison, as well as evaluating the combination of different effect size indices with RTs, (b) studying the power differential among alternative test statistic choices for RTs, and (c) constructing confidence intervals for effect sizes based on the RT rationale, and conducting a methodological and statistical evaluation of this method. 


\section{References}

Allison, D. B., \& Gorman, B. S. (1993). Calculating effect sizes for meta-analysis: The case of the single case. Behaviour Research and Therapy, 31, 621-631. doi:10.1016/00057967(93)90115-B

American Psychological Association (2010). Publication manual of the American Psychological Association (6th ed.). Washington, DC: American Psychological Association.

Barlow, D. H., Nock, M. K., \& Hersen, M. (2009). Single case experimental designs: Strategies for studying behavior change (3rd ed.). Boston, MA: Allyn \& Bacon.

Borckardt, J. J., Murphy, M. D., Nash, M. R., \& Shaw, D. (2004). An empirical examination of visual analysis procedures for clinical practice evaluation. Journal of Social Service Research, 30, 55-73. doi:10.1300/J079v30n03_04

Brossart, D. F., Parker, R. I., Olson, E. A., \& Mahadevan, L. (2006).The relationship between visual analysis and five statistical analyses in a simple $A B$ single-case research design. Behavior Modification, 30, 531-563. doi:10.1177/0145445503261167

Bulté, I., \& Onghena, P. (2008). An R package for single-case randomization tests. Behavior Research Methods, 40, 467-478. doi:10.3758/BRM.40.2.467

Bulté, I., \& Onghena, P. (2009). Randomization tests for multiple baseline designs: An extension of the SCRT-R package. Behavior Research Methods, 41, 477-485. doi:10.3758/BRM.41.2.477

Bulté, I., \& Onghena, P. (2012). When the truth hits you between the eyes. A software tool for the visual analysis of single-case experimental data. Methodology, 8, 104-114. doi:10.1027/1614-2241/a000042

Bulté, I., Van den Noortgate, W., \& Onghena, P. (2010, May). An R package for the nonparametric meta-analysis of small-n educational studies. Poster presented at the Annual Meeting of the American Educational Research Association (AERA), Denver, CO. Busk, P. L., \& Serlin, R. C. (1992). Meta-analysis for single-case research. In T. R. Kratochwill \& J. R. Levin (Eds.), Single-case research design and analysis: New directions for psychology and education (pp. 187-212). Hillsdale, NJ: Lawrence Erlbaum.

Campbell, J. M. (2003). Efficacy of behavioural interventions for reducing problem behaviour in persons with autism: A quantitative synthesis of single-subject research. Research in Developmental Disabilities, 24, 120-138. doi:10.1016/S0891-4222(03)00014-3

Campbell, J. M. (2004). Statistical comparison of four effect sizes for single-subject designs. Behavior Modification, 28, 234-246. doi:10.1177/0145445503259264 
Campbell, J. M. (2013). Commentary on PND at 25. Remedial and Special Education, 34, 2025. doi:10.1177/0741932512454725

Center, B. J., Skiba, R. J., \& Casey, A. (1985-1986). A methodology for the quantitative synthesis of intra-subject design research. Journal of Special Education, 19, 387-400. doi:10.1177/002246698501900404

Christ, T. J. (2007). Experimental control and threats to internal validity of concurrent and nonconcurrent multiple baseline designs. Psychology in the Schools, 44, 451-459. doi:10.1002/pits.20237

Crosbie, J. (1993). Interrupted time-series analysis with brief single-subject data. Journal of Consulting and Clinical Psychology, 61, 966-974. doi:10.1037/0022-006X.61.6.966

DeProspero, A., \& Cohen, S. (1979). Inconsistent visual analysis of intrasubject data. Journal of Applied Behavior Analysis, 12, 285-296. doi:10.1901/jaba.1979.12-573.

Dugard, P., File, P., \& Todman, J. (2012). Single-case and small-n experimental designs: A practical guide to randomization tests (2nd ed.). New York, NY: Routledge Academic.

Edgington, E. S. (1967). Statistical inference from N=1 experiments. Journal of Psychology, 65, 195-199. doi:10.1080/00223980.1967.10544864

Edgington, E. S. (1972). An additive method for combining probability values from independent experiments. Journal of Psychology, 80, 351-363. doi:10.1080/00223980.1972.9924813

Edgington, E. S. (1975). Randomization tests for one-subject operant experiments. Journal of Psychology, 90, 57-68. doi:10.1080/00223980.1975.9923926

Edgington, E. S. (1980). Overcoming obstacles to single-subject experimentation. Journal of Educational Statistics, 5, 261-267. doi:10.3102/10769986005003261

Edgington, E. S. (1984). Statistics and single case analysis. In M. Hersen, R. M. Eisler, \& P. M. Miller (Eds.), Progress in behavior modification: Vol. 16 (pp. 83-120). New York, NY: Raven Press.

Edgington, E., \& Onghena, P. (2007). Randomization tests (4th ed.). Boca Raton, FL: Chapman \& Hall/CRC.

Ernst, M. D. (2004). Permutation methods: A basis for exact inference. Statistical Science, 19, 676-685. doi:10.1214/088342304000000396

Ferron, J., \& Jones, P. K. (2006). Tests for the visual analysis of response-guided multiplebaseline data. Journal of Experimental Education, 75, 66-81. doi:10.3200/JEXE.75.1.66-81 Ferron, J., \& Onghena, P. (1996). The power of randomization tests for single-case phase designs. Journal of Experimental Education, 64, 231-239. 
Ferron, J., \& Sentovich, C. (2002). Statistical power of randomization tests used with multiple-baseline designs. Journal of Experimental Education, 70, 165-178. doi:10.1080/00220970209599504

Ferron, J., \& Ware, W. (1994).Using randomization tests with responsive single-case designs. Behaviour Research and Therapy, 32, 787-791. doi:10.1016/0005-7967(94)90037-X

Ferron, J., \& Ware, W. (1995). Analyzing single-case data: The power of randomization tests. Journal of Experimental Education, 63, 167-178. doi:10.1080/00220973.1995.9943820

Gage, N. A., \& Lewis, T. J. (2013). Analysis of effect for single-case design research. Journal of Applied Sport Psychology, 25, 46-60. doi:10.1080/10413200.2012.660673

Harbst, K. B., Ottenbacher, K. J., \& Harris, S. R. (1991). Interrater reliability of therapists' judgments of graphed data. Physical Therapy, 71, 107-115.

Hartmann, D. P., Gottman, J. H., Jones, R. R., Gardner, W., Kazdin, A. E., \& Vaught, R. S. (1980). Interrupted time-series analysis and its application to behavioral data. Journal of Applied Behavior Analysis, 13, 543-559. doi:10.1901/jaba.1980.13-543

Hedges, L. V., Cooper, H., \& Bushman, B. J. (1992). Testing the null hypothesis in metaanalysis: A comparison of combined probability and confidence interval procedures. Psychological Bulletin, 111, 188-194. doi:10.1037/0033-2909.111.1.188

Hedges, L. V., Pustejovsky, J. E., \& Shadish, W. R. (2012). A standardized mean difference effect size for single case designs. Research Synthesis Methods, 3, 224-239. doi:10.1002/jrsm.1052

Horowitz, M. J. (1982). Stress response syndromes and their treatment. In L. Goldberger \& S. Breznitz (Eds.), Handbook of stress: Theoretical and clinical aspects (pp. 711-732). New York, NY: Free Press.

Horowitz, M., Wilner, N., \& Alvarez, W. (1979). Impact of Event Scale: A measure of subjective stress. Psychosomatic Medicine, 41, 209-218.

Jones, R. R., Vaught, R. S., \& Weinrott, M. (1977). Time series analysis in operant research. Journal of Applied Behavior Analysis, 10, 151-166. doi:10.1901/jaba.1977.10-151

Joseph, S. (2000). Psychometric evaluation of Horowitz's impact of event scale: A review. Journal of Traumatic Stress, 13, 101-113. doi:10.1023/A:1007777032063

Kazdin, A. E. (1982). Single-case research designs: Methods for clinical and applied settings. New York, NY: Oxford University Press.

Kazdin, A. E. (1999). The meanings and measurement of clinical significance. Journal of Consulting and Clinical Psychology, 67, 332-339. doi:10.1037/0022-006X.67.3.332 
Kazdin, A. E. (2011). Single-case research designs: Methods for clinical and applied settings (2nd ed.). New York, NY: Oxford University Press.

Koehler, M. J. \& Levin, J. R. (1998). Regulated randomization: A potentially sharper analytical tool for the multiple-baseline design. Psychological Methods, 3, 206-217. doi:10.1037/1082-989X.3.2.206

Koehler, M. J., \& Levin, J. R. (2000). RegRand: Statistical software for the multiple-baseline design. Behavior Research Methods, Instruments, \& Computers, 32, 367-371. doi:10.3758/BF03207807

Kratochwill, T. R., Hitchcock, J., Horner, R. H., Levin, J. R., Odom, S. L., Rindskopf, D. M., \& Shadish, W. R. (2010). What Works Clearinghouse: Single-case designs technical documentation. Retrieved from http://ies.ed.gov/ncee/wwc/pdf/wwc_scd.pdf.

Kratochwill, T. R., \& Levin, J. R. (2010). Enhancing the scientific credibility of single-case intervention research: Randomization to the rescue. Psychological Methods, 15, 124-144. doi:10.1037/a0017736

Levin, J. R., Ferron, J. M., \& Kratochwill, T. R. (2012). Nonparametric statistical tests for single-case systematic and randomized $\mathrm{ABAB} . . . \mathrm{AB}$ and alternating treatment intervention designs: New developments, new directions. Journal of School Psychology, 50, 599-624. doi:10.1016/j.jsp.2012.05.001

Levin, J. R., Lall, V. F., \& Kratochwill, T. R. (2011). Extensions of a versatile randomization test for assessing single-case intervention effects. Journal of School Psychology, 49, 55-79. doi:10.1016/j.jsp.2010.09.002

Levin, J. R., \& Wampold, B. E. (1999). Generalized single-case randomization tests: Flexible analyses for a variety of situations. School Psychology Quarterly, 14, 59-93. doi:10.1037/h0088998

Maggin, D. M., Swaminathan, H., Rogers, H. J., O'Keeffe, B. V., Sugai, G., \& Horner, R. H. (2011). A generalized least squares regression approach for computing effect sizes in singlecase research: Application examples. Journal of School Psychology, 49, 301-321. doi:10.1016/j.jsp.2011.03.004

Manolov, R., Solanas, A., \& Leiva, D. (2010). Comparing "visual" effect size indices for single-case designs. Methodology, 6, 49-58. doi:10.1027/1614-2241/a000006

Manolov, R., Solanas, A., Bulté, I., \& Onghena, P. (2010). Data-division-specific robustness and power of randomization tests for ABAB designs. Journal of Experimental Education, 78, 191-214. doi:10.1080/00220970903292827 
Manolov, R., Solanas, A., Sierra, V., \& Evans, J. J. (2011). Choosing among techniques for quantifying single-case intervention effectiveness. Behavior Therapy, 42, 533-545. doi:10.1016/j.beth.2010.12.003

Matyas, T. A., \& Greenwood, K. M. (1990a). Visual analysis for single-case time series: Effects of variability, serial dependence, and magnitude of intervention effects. Journal of Applied Behavior Analysis, 23, 341-351. doi:10.1901/jaba.1990.23-341.

Matyas, T. A., \& Greenwood, K. M. (1990b). The effect of serial dependence on visual judgment of single-case charts: An addendum. American Journal of Occupational Therapy, 10, 308-320.

McKerracher, G., Powell, T., \& Oyebode, J. (2005). A single case experimental design comparing two memory notebook formats for a man with memory problems caused by traumatic brain injury. Neuropsychological Rehabilitation, 15, 115-128. doi:10.1080/09602010443000056

Nourbakhshs, M. R., \& Ottenbacher, K. J. (1994). The statistical analysis of single-subject data: A comparative examination. Physical Therapy, 74, 768-776.

Onghena, P. (1992). Randomization tests for extensions and variations of ABAB single-case experimental designs: A rejoinder. Behavioral Assessment, 14, 153-171.

Onghena, P. (2005). Single-case designs. In B. Everitt \& D. Howell (Eds.), Encyclopedia of statistics in behavioral science: Vol. 4 (pp. 1850-1854). New York, NY: Wiley.

Onghena, P., \& Edgington, E. S. (1994). Randomization tests for restricted alternating treatments designs. Behaviour Research and Therapy, 32, 783-786.

Onghena, P., \& Edgington, E. S. (2005). Customization of pain treatments: Single-case design and analysis. Clinical Journal of Pain, 21, 56-68. doi:10.1097/00002508-200501000-00007

Ottenbacher, K. J. (1990). When is a picture worth a thousand P-values? A comparison of visual and quantitative methods to analyze single subject data. Journal of Special Education, 23, 436-449. doi:10.1177/002246699002300407

Park, H., Marascuilo, L., \& Gaylord-Ross, R. (1990). Visual inspection and statistical analysis of single-case designs. Journal of Experimental Education, 58, 311-320.

Parker, R. I., \& Brossart, D. F. (2003). Evaluating single-case research data: A comparison of seven statistical methods. Behavior Therapy, 34, 189-211. doi:10.1016/S00057894(03)80013-8

Parker, R. I., \& Hagan-Burke, S. (2007). Useful effect size interpretations for single case research. Behavior Therapy, 38, 95-105. doi:10.1016/j.beth.2006.05.002 
Parker, R. I., Vannest, K. J., \& Davis, J. L. (2011). Effect size in single-case research: A review of nine nonoverlap techniques. Behavior Modification, 35, 303-322. doi:10.1177/0145445511399147

Perdices, M., \& Tate, R. L. (2009). Single-subject designs as a tool for evidence-based clinical practice: Are they unrecognised and undervalued? Neuropsychological Rehabilitation, 19, 904-927. doi:10.1080/09602010903040691

Pesarin, F. (2001). Multivariate permutation tests. New York, NY: Wiley.

Pesarin, F., \& Salmaso, L. (2010). Permutation tests for complex data: Theory, applications and software. Chichester, UK: Wiley.

Rasquin, S. M. C., van de Sande, P., Praamstra, A. J., \& Van Heugten, C. M. (2009). Cognitive-behavioural intervention for depression after stroke: Five single case studies on effects and feasibility. Neuropsychological Rehabilitation, 19, 208-222. doi:10.1080/09602010802091159

Rosenthal, R. (1978). Combining results of independent studies. Psychological Bulletin, 85, 185-193. doi:10.1037//0033-2909.85.1.185

Samuel, C., Louis-Dreyfus, A., Kaschel, R., Makiela, E., Troubat, M., Anselmi, N., Cannizzo, V., \& Azouvi, P. (2000). Rehabilitation of very severe unilateral neglect by visuo-spatiomotor cueing: Two single case studies. Neuropsychological Rehabilitation, 10, 385-399.

Schulz, R., O'Brien, A., Czaja, S., Ory, M., Norris, R., Martire, L. M., et al. (2002). Dementia caregiver intervention research: In search of clinical significance. Gerontologist, 42, 589-602. Scruggs, T. E., \& Mastropieri, M. A. (2013). PND at 25: Past, present, and future trends in summarizing single-subject research. Remedial and Special Education, 34, 9-19. doi:10.1177/0741932512440730

Scruggs, T. E., Mastropieri, M. A., \& Casto, G. (1987). The quantitative synthesis of singlesubject research. Methodology and validation. Remedial and Special Education, 8, 24-33. doi:10.1177/074193258700800206

Scruggs, T. E., Mastropieri, M. A., Cook, S. B., \& Escobar, C. (1986). Early interventions for children with conduct disorders: A quantitative synthesis of single-subject research. Behavioral Disorders, 11, 260-271.

Shadish, W. R., Rindskopf, D. M., \& Hedges, L. V. (2008). The state of the science in the meta-analysis of single-case experimental designs. Evidence-Based Communication Assessment and Intervention, 2, 188-196. doi:10.1080/17489530802581603 
Shakespeare, T. P., Gebski, V. J., Veness, M. J., \& Simes, J. (2001). Improving interpretation of clinical studies by use of confidence levels, clinical significance curves, and risk-benefit contours. Lancet, 357, 1349-1353. doi:10.1016/S0140-6736(00)04522-0

Task Force on Evidence-Based Interventions in School Psychology (2003). Procedural and coding manual for review of evidence-based interventions. Retrieved from http://www.indiana.edu/ ebi/documents/_workingfiles/EBImanual1.pdf

Todman, J. B., \& Dugard, P. (2001). Single-case and small-n experimental designs: A practical guide to randomization tests. Mahwah, NJ: Erlbaum.

Van den Noortgate, W., \& Onghena, P. (2003a). Combining single-case experimental data using hierarchical linear models. School Psychology Quarterly, 18, 325-346. doi:10.1521/scpq.18.3.325.22577

Van den Noortgate, W., \& Onghena, P. (2003b). Hierarchical linear models for the quantitative integration of effects sizes in single-case research. Behavior Research Methods, Instruments, \& Computers, 35, 1-10. doi:10.3758/BF03195492

Van den Noortgate, W., \& Onghena, P. (2008). A multilevel meta-analysis of single-subject experimental design studies. Evidence-based Communication Assessment and Intervention, 2, 142-151. doi:10.1080/17489530802505362

Wolery, M., Busick, M., Reichow, B., \& Barton, E. E. (2010). Comparison of overlap methods for quantitatively synthesizing single-subject data. Journal of Special Education, 44, 18-29. doi:10.1177/0022466908328009 
Figure 1. Graphical display of the hypothetical data set: A randomized single-case ABAB phase design.

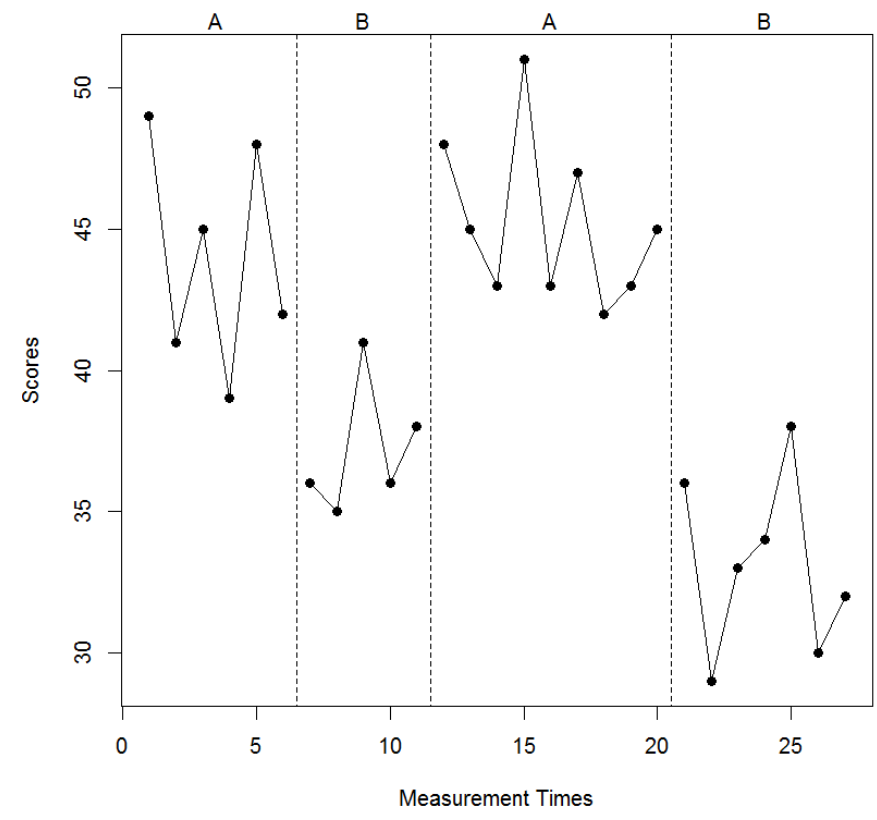


Table 1

Hypothetical Data Set: A Randomized Single-Case ABAB Phase Design

\begin{tabular}{llllllllllll}
\hline Treatment & A1 & A1 & A1 & A1 & A1 & A1 & B1 & B1 & B1 & B1 & B1 \\
Score & 49 & 41 & 45 & 39 & 48 & 42 & 36 & 35 & 41 & 36 & 38 \\
\hline
\end{tabular}

\begin{tabular}{lllllllllllllllll}
\hline Treatment & A2 & A2 & A2 & A2 & A2 & A2 & A2 & A2 & A2 & B2 & B2 & B2 & B2 & B2 & B2 & B2 \\
Score & 48 & 45 & 43 & 51 & 43 & 47 & 42 & 43 & 45 & 36 & 29 & 33 & 34 & 38 & 30 & 32 \\
& & & & & & & & & & & & & & & & \\
\hline
\end{tabular}

